\title{
A Numerical Study for Turbulent Flow and Thermal Influence over Inhomogenous Canopy of Roughness Elements
}

\author{
ZHENGTONG XIE* and JIACHUN LI ${ }^{\mathrm{a}}$ \\ School of Engineering Sciences, University of Southampton, Southampton SO17 1BJ, UK \\ anstitute of Mechanics, Chinese Academy of Sciences, Beijing 100080, China
}

Received 13 April 2005; accepted in revised form 22 August 2005

\begin{abstract}
A large-eddy simulation with transitional structure function(TSF) subgrid model we previously proposed was performed to investigate the turbulent flow with thermal influence over an inhomogeneous canopy, which was represented as alternative large and small roughness elements. The aerodynamic and thermodynamic effects of the presence of a layer of large roughness elements were modelled by adding a drag term to the threedimensional Navier-Stokes equations and a heat source/sink term to the scalar equation, respectively. The layer of small roughness elements was simply treated using the method as described in paper (Moeng 1984, J. Atmos Sci. 41, 2052-2062) for homogeneous rough surface. The horizontally averaged statistics such as mean vertical profiles of wind velocity, air temperature, et al., are in reasonable agreement with Gao et al. (1989, Boundary layer meteorol. 47, 349-377) field observation (homogeneous canopy). Not surprisingly, the calculated instantaneous velocity and temperature fields show that the roughness elements considerably changed the turbulent structure within the canopy. The adjustment of the mean vertical profiles of velocity and temperature was studied, which was found qualitatively comparable with Belcher et al. (2003, J Fluid Mech. 488, 369-398)'s theoretical results. The urban heat island(UHI) was investigated imposing heat source in the region of large roughness elements. An elevated inversion layer, a phenomenon often observed in the urban area (Sang et al., J Wind Eng. Ind. Aesodyn. 87, 243-258)'s was successfully simulated above the canopy. The cool island(CI) was also investigated imposing heat sink to simply model the evaporation of plant canopy. An inversion layer was found very stable and robust within the canopy.
\end{abstract}

Key words: canopy, cool island, inhomogeneous, large-eddy simulation, roughness element, urban heat island

\section{Introduction}

The heat, mass and momentum transfer in atmospheric boundary layer (ABL) over inhomogeneous underlying surface is of fundamental and practical importance. (1) An urban area (city scale, see [2]) is composed of

\footnotetext{
${ }^{*}$ Corresponding author, E-mail: z.xie@soton.ac.uk
} 
buildings, streets, parks; (2) a metropolis or a densely populated area (regional scale, see [2]), such as Yangtz River Delta, Pear River Delta, Greater London, commonly has a centre city surrounded with satellite towns, and patches of rural areas; (3) in arid and semiarid areas, e.g. in northwest China. Oasis - desert area and patches of agricultural fields separated with dry bare soil, are very sensitive to environmental change; (4) windbreak is widely used in north China, e.g. "SAN BEI" windbreak, to protect crops, to reduce soil erosion and to prevent desertification; (5) the general circulation of atmosphere model (GCM) relies heavily on the grid parameterization. The grid square is no less than about $10 \mathrm{~km}$ on a side in GCM. There always scatter farmland, forests, grasslands, lakes, bogs, cities/towns et al. on a single grid. However, the understanding of the mechanism by which the urban boundary layer and the rural boundary layer, the atmospheric surface layer and a plant canopy are coupled aerodynamically and thermodynamically is still in its infancy.

Belcher et al. [1] developed a model for the adjustment of the spatially averaged time-mean flow of a deep turbulent boundary layer over small roughness elements to a canopy of larger three-dimensional roughness elements. They found that their general approach can also be applied to the changes in scalar fields associated with large roughness changes. But again thermal effects, e.g. thermal buoyancy, has not yet been considered in this model. Martilli [20] used a two-dimensional regional scale model with detailed urban surface exchange parameterization to study the urban influences (mainly mechanical and thermal factors) on boundary layer structures.

However, because of the complex physical processes involved in the atmospheric surface layer and the canopy, it is probably impossible to develop theoretically a general and yet simple ensemble-mean turbulence model for climate and environment applications [22, 23]. When Reynolds Averaged Navie-Stokes (RANS) equation method is used, it is implicitly assumed that there is a fair degree of scale separation between the large time scale of the unsteady flow features and the time scale of the genuine turbulence [3]. However, in reality it is hard to find an evident time scale gap for most turbulent flows. And, of course, RANS generally eliminates most of the genuinely turbulent fluctuation information.

Large-eddy simulation (LES) [28] is a promising tool for computing unsteady three-dimensional flows at high Reynolds number or with complex geometry. An LES resolves only the large-scale fluid motions and models the subgrid-scale (SGS) motions through filtering the NavierStokes equations (see Equations 1-3). Since Smagorinsky and Deardorff's pioneering work, scientists have carried out LES for the investigation of ABL [12, 22, 30, 34]. Most of the researches focused on atmospheric boundary layer ABL turbulent flows over homogeneous surface with/without plant canopy. 
Patton et al. [25] performed a LES of turbulent flow around multiple windbreaks set within a wheat canopy under neutral stability conditions. Recently, turbulent flows over multiple cubes (staggered or aligned) are calculated using LES by several groups $[5,13,31,36]$ to investigate urban canopy flows. Again, these numerical simulations are all under neutral stability conditions.

Coherent structures are observed in various turbulent flows. In the wall region of boundary layer, ejection-sweep cycles have been commonly observed through flow visualization techniques [14, 32]. The coherent structures are the most efficient for transfer of momentum, mass and heat. Gao et al. (1989) observed ramp patterns of temperature and humidity composed of weak ejecting motions transporting warm and/or moist air out of the forest followed by strong sweeps of cool and/or dry air penetrating into the canopy. However, so far very little is known about the coherent structures in an inhomogeneous canopy.

Actually, it is unnecessary to resolve all the leaves, branches and trunks of a plant canopy. Furthermore,it is possible but very expensive to resolve all the buildings in a urban canopy using LES [5, 13, 20, 31, 36]. Therefore, the aerodynamic and thermodynamic effects of the presence of a canopy are commonly expressed by adding a drag term to the three-dimensional Navier-Stokes equations and a heat source/sink term to the thermodynamic equation. Modern urban area with high buildings can be simplified as such a canopy if we are not interested in the details in the street scale. One of the main issues which we have to confront is the evaluation of surface fluxes over non-homogeneous terrain [17] for GCM. The above method can be used to improve the grid parameterization of GCM.

In the current paper, the large eddy simulation governing equations, the TSF SGS model and the canopy model were extensively examined at first. Second, an LES was validated by comparing the calculated turbulent flow over a homogeneous canopy with field observation. Third, the turbulent flow with thermal influence over an area alternately covered with large roughness elements (plant canopy) and small roughness elements (bair soil) was simulated. And we finally come to a number of enlightening conclusions.

\section{The Large Eddy Simulation}

\subsection{THE GOVERNING EQUATIONS}

The equations of motion for resolved-scale velocity are given as follows,

$$
\frac{\partial \bar{u}}{\partial t}=\overline{\bar{v} \bar{\zeta}_{z}}-\overline{\bar{w} \bar{\zeta}_{y}}-\frac{\partial P^{*}}{\partial x}-\frac{\partial\langle\bar{p}\rangle}{\partial x}-\frac{\partial \tau_{x x}}{\partial x}-\frac{\partial \tau_{x y}}{\partial y}-\frac{\partial \tau_{x z}}{\partial z}+F_{x},
$$




$$
\begin{aligned}
& \frac{\partial \bar{v}}{\partial t}=\overline{\bar{w} \bar{\zeta}_{x}}-\overline{\bar{u} \bar{\zeta}_{z}}-\frac{\partial P^{*}}{\partial y}-\frac{\partial\langle\bar{p}\rangle}{\partial y}-\frac{\partial \tau_{x y}}{\partial x}-\frac{\partial \tau_{y y}}{\partial y}-\frac{\partial \tau_{y z}}{\partial z}+F_{y}, \\
& \frac{\partial \bar{w}}{\partial t}=\overline{\bar{u} \bar{\zeta}_{y}}-\overline{\bar{v} \bar{\zeta}_{x}}-\frac{g \bar{\theta}}{\theta_{0}}-\frac{\partial P^{*}}{\partial z}-\frac{\partial \tau_{x z}}{\partial x}-\frac{\partial \tau_{y z}}{\partial y}-\frac{\partial \tau_{z z}}{\partial z}+F_{z},
\end{aligned}
$$

where the overbar denotes the resolved-scale field, $F_{i}$ is the drag force of plant canopy, while over bare soil these terms vanish. $g$ the gravity acceleration, $\zeta_{i}$ the vorticity component in $i$ direction, and $\tau$ are the SGS Reynolds stresses. The SGS term will be described in detail in Section 2.2. And,

$$
P^{*}=\frac{\bar{p}}{\rho}+\frac{R_{k k}}{3}+\frac{\overline{\bar{u}_{k} \bar{u}_{k}}}{2} .
$$

The SGS stresses $\tau$ are defined as,

$$
\tau_{i j}=R_{i j}-R_{k k} \delta_{i j} / 3
$$

where

$$
R_{i j}=\overline{u_{i}^{\prime} u_{j}^{\prime}}+\overline{u_{i}^{\prime} \bar{u}_{j}}+\overline{\bar{u}_{i} u_{j}^{\prime}} .
$$

The continuity equation is written as,

$$
\frac{\partial \bar{u}}{\partial x}+\frac{\partial \bar{v}}{\partial y}+\frac{\partial \bar{w}}{\partial z}=0
$$

Using Equations (1)-(3) and (7), we get a Poisson equation to solve the pressure field.

$$
\nabla^{2} P^{*}=\frac{\partial H_{x}}{\partial x}+\frac{\partial H_{y}}{\partial y}+\frac{\partial H_{z}}{\partial z}
$$

where $H_{x}, H_{y}$ and $H_{z}$ are the sums of the right-hand sides of Equations $1-3$.

The thermodynamic equation is

$$
\frac{\partial \bar{\theta}}{\partial t}=-\bar{u} \overline{\frac{\partial \bar{\theta}}{\partial x}}-\bar{v} \overline{\frac{\partial \bar{\theta}}{\partial y}}-\overline{\bar{w} \frac{\partial \bar{\theta}}{\partial z}}-\frac{\partial \tau_{\theta x}}{\partial x}-\frac{\partial \tau_{\theta y}}{\partial y}-\frac{\partial \tau_{\theta z}}{\partial z}+S,
$$

here $\bar{\theta}$ is the resolved-scale potential temperature. $\tau_{i \theta}$ the SGS heat flux, $S$ is heat source/sink of plant canopy which will be described in detail in Section 2.3.

The SGS kinetic energy equation is written as follows,

$$
\frac{\partial \overline{e^{\prime}}}{\partial t}=-\overline{\bar{u}_{i} \frac{\partial \overline{e^{\prime}}}{\partial x_{i}}}-\overline{u_{i}^{\prime} u_{j}^{\prime}} \frac{\partial \overline{u_{i}}}{\partial x_{j}}+\frac{g}{\theta_{0}} \overline{w^{\prime} \theta^{\prime}}-\frac{\partial \overline{\left[u_{i}^{\prime}\left(e^{\prime}+p^{\prime} / \rho_{0}\right)\right]}}{\partial x_{i}}-\epsilon-2 \frac{\overline{e^{\prime}}}{\tau},
$$


where $\epsilon$ is the dissipation rate; the last term models the effect of the canopy layer, $\tau$ is a time scale for the drag of canopy to be defined in Section 2.3 .

\subsection{SGS MODEL}

The SGS model plays a critical role for the ABL flows at very high Reynolds number. The issue on SGS model still remains. The SGS models can be classified into three groups: (1) eddy viscosity model, (2) similarity model and (3) mixed model. Metais and Lesieur [21] (1996) proposed a structure-function (SF) model, aiming at taking into account of the local intermittence and underdevelopment of small scale motion to reduce excessive dissipation. The model gives better results for free and wall shear flows, separated flows [16]. To our knowledge, so far SF model has not yet been applied in the convective atmospheric boundary layer. The SF model is an eddy viscosity model. The similarity model can simulate the so called "backscatter", but it under-estimates the turbulent diffusion. The mixed model $[11,37]$ attempts to overcome the defects of the other models. But the mixed models are much more complicated to use.

Among these studies [6, 22, 24], the "turbulent energy model" is commonly used for ABL flows. The model combines a characteristic length scale(the grid-cell size) and a characteristic velocity scale (the square root of the SGS turbulent kinetic energy) for the highly inhomogeneous turbulence in which an equilibrium range is not well developed instantaneously. The model is written as follows,

$$
\tau_{i j}=-v_{e}\left(\frac{\partial \overline{u_{i}}}{\partial x_{j}}+\frac{\partial \overline{u_{j}}}{\partial x_{i}}\right),
$$

where the eddy diffusivity $v_{e}$ is assumed to be proportional to a SGS velocity scale $\left({\overline{e^{\prime}}}^{1 / 2}\right)$ and a characteristic length $l$,

$$
v_{e}=0.1 l \bar{e}^{1 / 2} \text {. }
$$

However, the above SGS model exhibits unsatisfactory performance in the vicinity of rigid surface or the top of plant canopy [15].

Metais and Lesieur [21] (1996) applied the spectral eddy-viscosity in physical space and proposed a SF model. The following is a brief description of the SF model,

$$
v_{s}=0.105 C_{k}^{-3 / 2} \Delta * F(\vec{x}, \Delta)^{1 / 2},
$$

where $\Delta$ is the scale of subgrid, and $C_{k}=1.4, F$ is the second-order structure function of the resolved velocity field. $F$ is calculated with a local 
statistical average of square velocity differences between the computational grid at $\vec{x}$ and the six closest surrounding grid points,

$$
F(\vec{x}, \Delta)=\frac{1}{6} \sum_{i=1}^{3}\left[\left\|\vec{u}(\vec{x})-\vec{u}\left(\vec{x}+\Delta x_{i} \vec{e}_{i}\right)\right\|^{2}+\left(\left\|\vec{u}(\vec{x})-\vec{u}\left(\vec{x}-\Delta x_{i} \vec{e}_{i}\right)\right\|^{2}\right] .\right.
$$

Since the ABL flows are high Reynolds number turbulent flows, it is appropriated to apply the SGS TKE equation (10) to determine the velocity scale for the eddy viscosity for weak convective ABL flows. There exists a strong shear layer in the vicinity of canopy top, where the eddy scale decreases approaching the canopy. How to capture the shear layer is the key to simulate such flows successfully. Therefore, the SGS model should capture both the local intermittence and the anisotropy characteristics for shear-driven boundary layers. In our previous paper [18], we proposed a new SGS model, which is called transitional structure function (TSF) model. The TSF model is a combination of turbulent energy and SF model, to account for the strong shear layer and the instability of ABL flow at high Reynolds number. The TSF model is an eddy viscosity model (see equation, where the eddy viscosity $v_{t}$ consists of two parts: the turbulent energy part $\beta * v_{e}$ (see Equation (12)) and the SF part $(1-\beta) * v_{s}$ (see Equation (13)),

$$
v_{t}=\beta * v_{e}+(1-\beta) * v_{s},
$$

where $\beta$ is a weighting factor ranging from 0 (on the top of canopy) to 1 (on the top of domain). Here, $\beta$ is simply taken as a linear function of $z$. The TSF model can be considered as a simplified dynamic model.

\subsection{CANOPY DESCRIPTION}

The full details of air flow within and above plant canopy/urban canopy is too complicated to compute. One simple way is that it is considered as horizontally homogeneous source/sink, while the leaf area density is vertically distributed,

$$
F_{i}=C_{d} a V \overline{u_{i}}=-\bar{u}_{i} / \tau,
$$

where $C_{d}=0.15$ is homogeneous resistance coefficient [30], $a$ the leaf area density $\left(\mathrm{m}^{-1}\right)$ at vertical lever $z, V=\left(\bar{u}_{i} \bar{u}_{i}\right)^{1 / 2}$ is the magnitude of velocity.

Instead of solving an energy balance equation between the plant leaves and the ambient air flow, e.g. in our previous work using RANS [33], the heat source/sink was simply vertically distributed within the canopy. It is because that solving the nonlinear energy balance equation makes the computation much more expensive. The term $S$ in Equation(9) is defined by the vertical derivative of the heat flux given as, 
Table I. Numerical settings.

\begin{tabular}{llllll}
\hline $\begin{array}{l}\mathrm{U}(\mathrm{m} / \mathrm{s}) \\
(\text { at } 80 \mathrm{~m})\end{array}$ & $\begin{array}{l}u_{*}(\mathrm{~m} / \mathrm{s}) \\
\text { (at 20m) }\end{array}$ & $\begin{array}{l}Q_{*}\left(\mathrm{~ms}^{-1} \mathrm{~K}\right) \\
\text { (heat flux } \\
\text { from canopy }\end{array}$ & $\begin{array}{l}Q_{s}\left(\mathrm{~ms}^{-1} \mathrm{~K}\right) \\
\text { (heat flux } \\
\text { from surface }\end{array}$ & $\begin{array}{l}z_{0}(\mathrm{~m}) \\
\text { (surface } \\
\text { roughness height) }\end{array}$ & $\begin{array}{l}\mathrm{L}(\mathrm{m}) \\
\text { (Monin- } \\
\text { Obukhov } \\
\text { length) }\end{array}$ \\
\hline 3 & 0.46 & 0.084 & 0.016 & 0.016 & -87 \\
\hline$(z)=Q(h) \exp (-\alpha F), \quad F(z)=\int_{z}^{h} a \mathrm{~d} z$, &
\end{tabular}

where $Q(h)$ is the total radiation flux, $Q(z)$ the radiation flux at lever $z$ within canopy, $F(z)$ is the summation of leaf area above lever $z$ and $\alpha$ is the attenuation factor.

\subsection{BOUNDARY CONDITIONS}

Periodic boundary condition was adopted in the horizontal direction. A constant pressure gradient was used as the the driving force of the flow. In the vertical direction, the slipping boundary condition was applied at the top border, while the bottom boundary condition was chosen to match the law of wall at the first grid point over the surface. In particular, the Businger-Dyer formula was used to take account of the stability of the atmosphere [22]. Note the heat flux on the bottom surface was much less than the canopy heat flux, which is shown in Table I. Also, a wall model proposed by Wyngaard was used to improve the prediction the near-wall fluctuations for the rough wall $[22,34]$.

\section{Some Results of Homogenous Canopy}

To validate the LES for plant canopy flow, we performed an LES for turbulent flow above and within a plant canopy. The computational domain was $L_{x} \times L_{y} \times L_{z}=9.6 \mathrm{~h} \times 9.6 \mathrm{~h} \times 3.2 \mathrm{~h}$, where the height $h$ of forest canopy is $20 \mathrm{~m}$. Although this domain with periodic boundary conditions turns out not to be able to capture all of the large scales of turbulence, most of the turbulent kinetic energy is obtained [36]. A uniform mesh with the grid number $n_{x} \times n_{y} \times n_{z}=96 \times 96 \times 32$ was used. No doubt this is a very coarse mesh with only 10 grids per plant height. However, it was found [36] that a uniform mesh with only eight grid points per cube height yielded reasonable results. We can not afford more grids per plant height if we want to simulate the whole ABL with approximately $1000 \mathrm{~m}$ depth.

The LES code solved time-dependent, incompressible Navier-Stokes equations. A spectral method was used for horizontal spatial derivatives, while a centered finite difference method with a vertically staggered grid 
is used in the vertical direction. All quantities were advanced in time by Adams-Bathforth scheme with the time step $0.1 \mathrm{~s}$. It took $11 \mathrm{~h} \mathrm{CPU}$ time of SGI Origin 2000 parallel supercomputer to advance 6400 time steps. A test case of weak convective condition with Monin-Obokov length $L=$ $-700 \mathrm{~m}$ (with $\mathrm{LAI}=5, \mathrm{LAI}=2$, respectively) was simulated.

Figure 1(left) shows the vertical profiles of leaf area density $\left(\mathrm{m}^{-1}\right)$, where the leaf area index (LAI) is 5 and 2, respectively [30]. Figure 1 (right) shows the vertical profiles of normalized heat source within the canopy. Note the height of maximum heat source is approximately at the height $\mathrm{z} / \mathrm{h}=0.8$, which does not coincide with that $(\mathrm{z} / \mathrm{h} \simeq 0.6)$ of the leaf area density. The sum of the heat flux imposed into air is $0.40 Q(h)$ for $\mathrm{LAI}=5$, $0.33 Q(h)$ for $\mathrm{LAI}=2$, respectively.

Figure 2 shows the vertical profiles of streamwise velocity, Reynolds stress and turbulent kinetic energy. Figure $2 \mathrm{a}$ and $\mathrm{b}$ show the calculated vertical profiles of averaged streamwise velocity for LAI 5 and 2, respectively, the observed data of Gao et al. [10] and LES results of Shaw \& Schumann [30]. Gao's experiment was conducted at Camp Borden, Ontario, Canada in August, 1987, with full summer foliage of LAI=1.9. The forest, of approximate mean height $18 \mathrm{~m}$, was composed primarily of aspen and maple. Note all the data are normalized by the free stream velocity. The comparison is reasonable. The profiles show near-logarithmic shape above the forest and near-exponential decay in the upper half of the forest. Both profiles in Figure 2a show a reversal of the velocity gradient in the lower half of the canopy. But the feature is not found in Figure 2b, which is due to the less leaf area density.
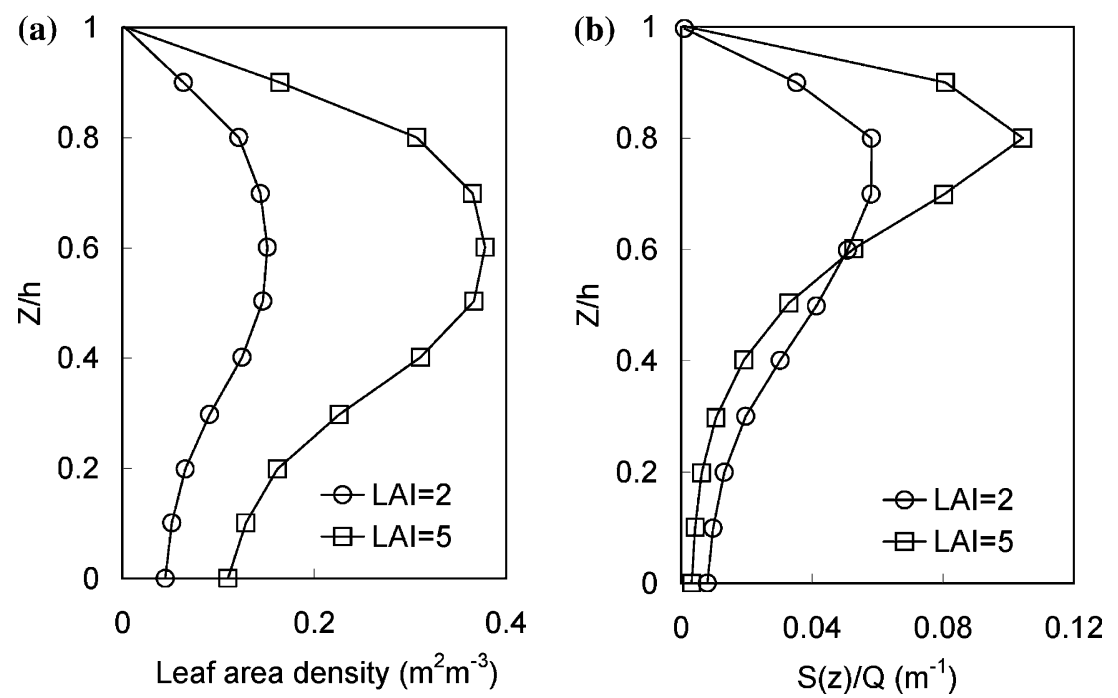

Figure 1. Vertical distribution of leaf area density (left) and heat sources (right). 
(a)

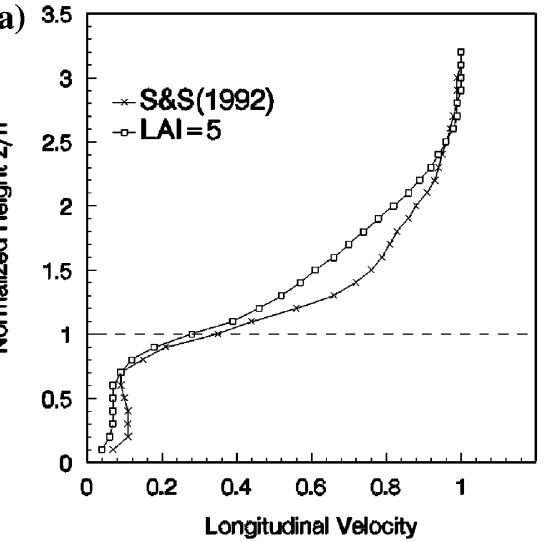

(c)
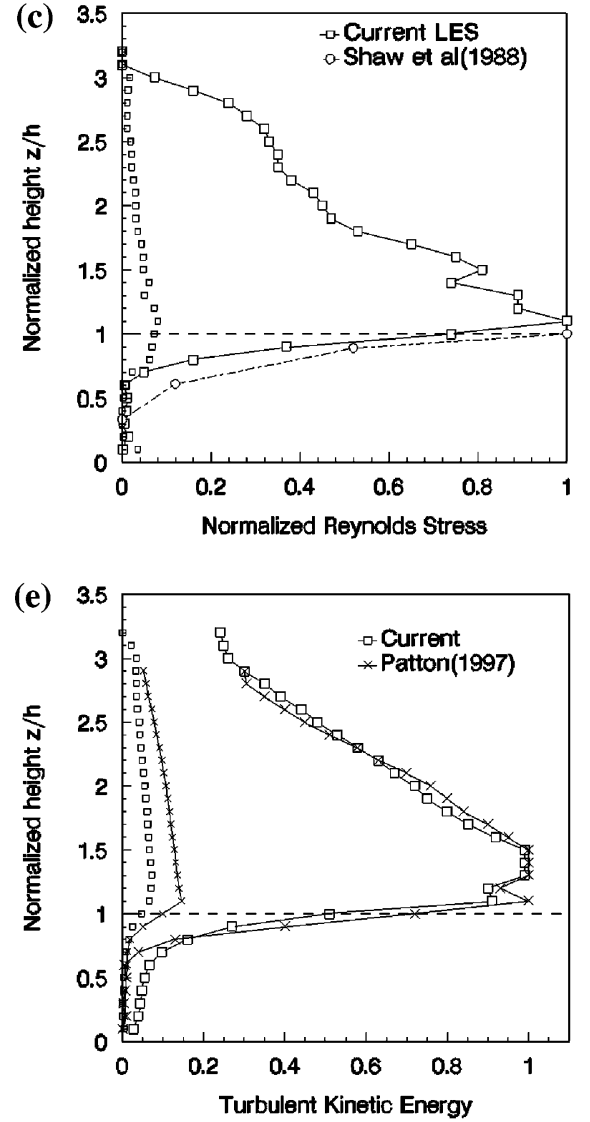
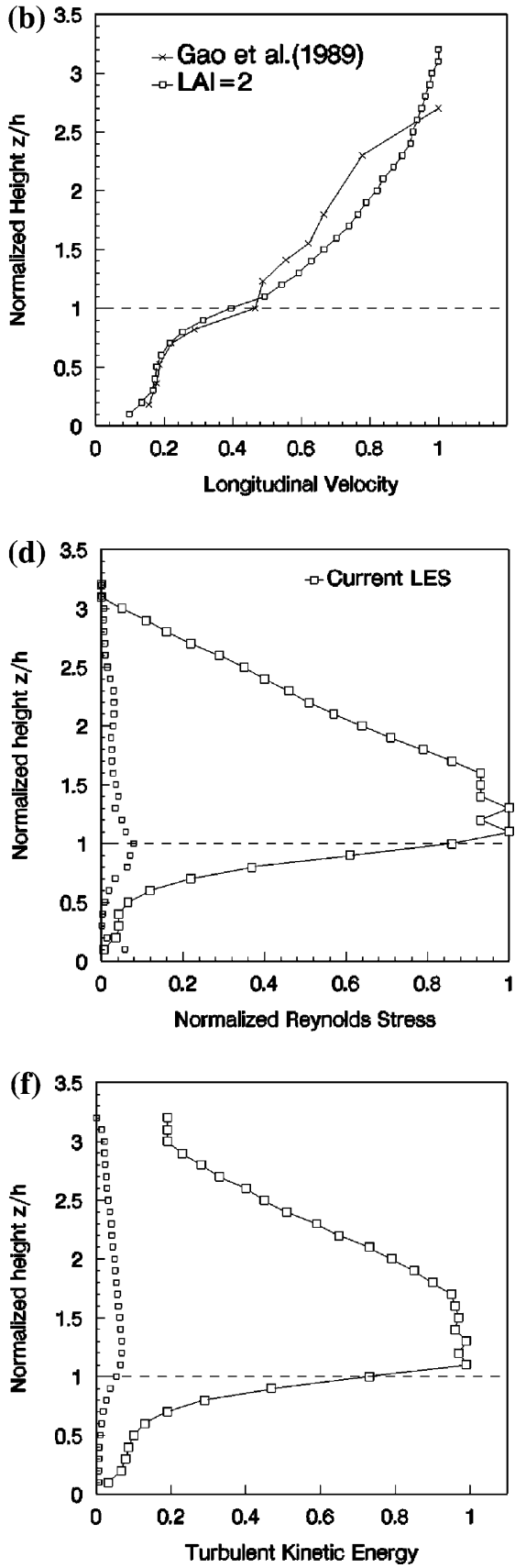

Figure 2. Vertical profiles of streamwise velocity, Reynolds stress, turbulent kinetic energy. Left, $\mathrm{LAI}=5$, right, $\mathrm{LAI}=2$. 
Figure 2c illustrates the vertical profiles of Reynolds shear stress (normalized by the data at the canopy height) obtained from the current LES for $\mathrm{LAI}=5$ and the measured data of Shaw et al.[29], where the large squares are the total Reynolds stress and the small ones are the SGS component. The SGS component is fairly small, less than $8 \%$ of the total. Note the peak of the vertical profile of normalized Reynolds stress is located approximately at the height of canopy. The Reynolds stress decreases rapidly within the canopy. Figure $2 \mathrm{~d}$ shows the vertical profiles of normalized Reynolds stress obtained from the current LES for $\mathrm{LAI}=2$, which are quite similar in shape to those in Figure 2c in qualitative sense.

Figure $2 \mathrm{e}$ presents the vertical profiles of total normalized turbulent kinetic energy and the SGS component for $\mathrm{LAI}=5$, where the squares are current LES results, the crosses are LES results of Patton [24] and the smaller symbols are SGS components. The SGS component is about $5 \%$ of the total in the current LES, but more than 10\% in Patton's LES results where the turbulent energy model was used. The TKE and the SGS component in Figure 2e show that the TSF SGS model results in a reduction of the SGS component, which suggests the results generated by current LES are less sensitive to the quality of the SGS model [7]. Figure $2 f$ depicts the vertical profiles of total normalized turbulent kinetic energy and the SGS component as in figure $2 \mathrm{e}$ but for $\mathrm{LAI}=2$.

The vertical cross-sections of velocity and temperature for weak unstable condition were also investigated. No temperature ramp was found, which might be because that the temperature gradient was too weak for the weak convective condition. However, we did observed some events, e.g. a fairly strong ejecting motion transporting warm air out of canopy followed by a weak sweep of cool air penetrating into the canopy. Since we did not focus much on coherent structures in this research, only a very small dataset was analyzed. We might not have captured a full spectrum of the structures. It is worth noting that in the field experiment [10], it was found that the sweep contributes much more to the mean fluxes of momentum than the ejection. Gao et al. concluded that in general a coherent structure consists of a weak ejection from the canopy top followed by a strong sweep into the canopy. However, it was found in the large eddy simulations [9, 12] that the contribution of ejection to the momentum fluxes is comparable to that of sweep. We speculate that in the field experiment there existed very large scale eddies above the canopy, whereas in the LES the smaller domain size restricts the large eddies above the canopy, which consequently makes the sweep motion weaker. Overall, the TSF SGS model, the canopy model and the numerical settings were successful, which suggests us to use these to simulate flows over inhomogenous canopy. 


\section{Some Results of Inhomogenous Canopy}

An abridged general view of the domain is shown in Figure 3. The computational domain was $L_{x} \times L_{y} \times L_{z}=192 \mathrm{~m} \times 192 \mathrm{~m} \times 80 \mathrm{~m}$, where the plant canopy (large roughness elements) covered $96 \mathrm{~m}$ in $x$ direction, the rest was bare soil (small roughness elements). The height of plant canopy was $20 \mathrm{~m}$ with $\mathrm{LAI}=5$. A uniform mesh with the grid number $n_{x} \times n_{y} \times n_{z}=32 \times$ $32 \times 40$ was used. Table 2.4 shows the numerical settings for the LES. Note $Q_{*}$ is the total imposed heat flux integrated through the canopy depth, whereas $Q(h)$ in Equation (17) is $0.213 \mathrm{~ms}^{-1} \mathrm{~K}$. We averaged 100 time steps and also averaged in spanwise direction to calculate the mean field, where the time step is $0.2 \mathrm{~s}$.

Figure 4 shows the vertical profiles of streamwise velocity over inhomogeneous canopy vs. those over homogeneous canopy. The plot for 'over bare soil of inhomogeneous canopy' is the data spatially averaged in the streamwise direction and the spanwise direction over the whole region of bare soil. The plot for 'over plant of inhomogeneous canopy' is the averaged data over the whole region of plant. The plot labelled as 'inhomogeneous canopy' is a horizontal average over the whole domain. Significant difference of the inhomogeneous canopy flow is found between the bare soil area and plant area below height $\mathrm{Z} / \mathrm{h}=1$. Note that the streamwise velocity in the plant region is larger than that in the bare soil region below the height $\mathrm{Z} / \mathrm{h}=1$, which is mainly due to a large clockwise circulation in the bare soil region and the shielding of the plant canopy (also see Figure 5). However, the streamvise velocity over inhomogeneous canopy with horizontally averaged LAI $=2.5$ is in reasonable agreement with those over homogeneous canopy, of which LAI is 1.9 and 2.0 for Gao et al.'s

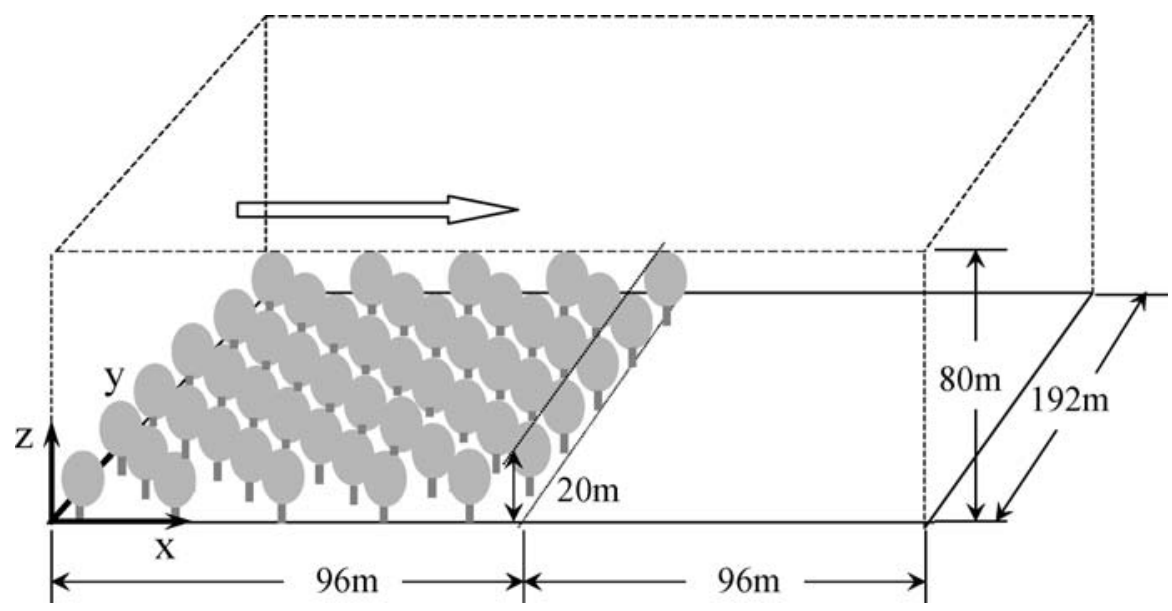

Figure 3. Abridged general view of the domain. 


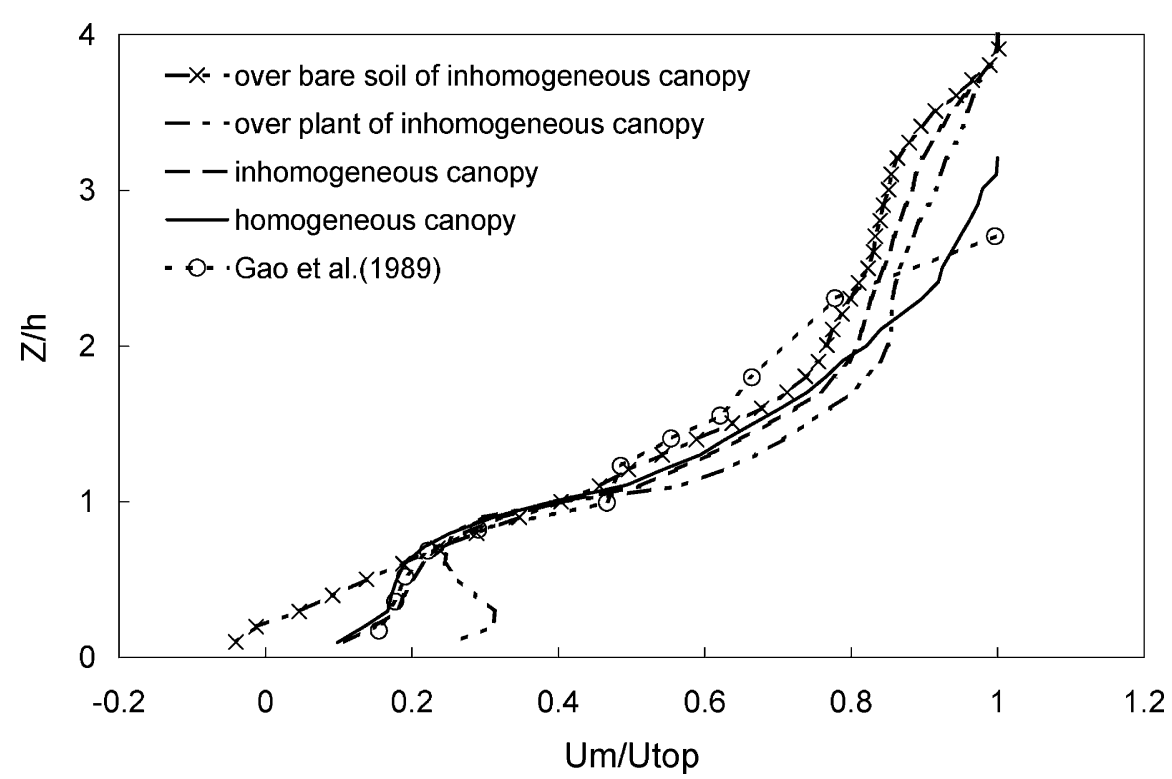

Figure 4. Profiles of streamwise velocity over inhomogeneous canopy vs. those over homogeneous canopy.

field measurements and our LES data, respectively. This might suggest that the horizontally averaged velocity is not sensitive to the inhomogeneity of canopy. However, the current case might be the simplest 'inhomogeneity', and only one domain setup was used. More investigations, for instance, a different domain setup, an inhomogeneity in spanwise direction, etc, are needed to confirm the suggestion. Also note that it might be too ambitious to extend the tentative suggestion to the inhomogeneity of the arrangement of roughness elements. In the current distributed drag approach, a constant resistance coefficient of the canopy was used for simplicity (see Equation (16)). Whereas in the real situation of roughness elements, the resistance coefficient depends on the arrangement of the obstacles. It would be very helpful to incorporate into the drag parameterisation a suitable dependence on some typical arrangements of roughness elements.

Figure 5 shows four typical instantaneous visualizations of the vertical cross-section of velocity vector field at $y=\frac{1}{2} L_{y}$. Distinct clockwise circulations were observed very frequently in the bare soil area, while these circulations are fairly weak. On the contrary, the upward forcing on the flow at the leading edge of plant canopy is quite evident. The upward flows at the leading edge of plant canopy and the weak clockwise circulations in the bare soil area, which are the shielding of the canopy, may explain that the horizontally averaged streamwise velocity within the plant canopy is larger than that at the same height in the bare soil area in Figure 4. 

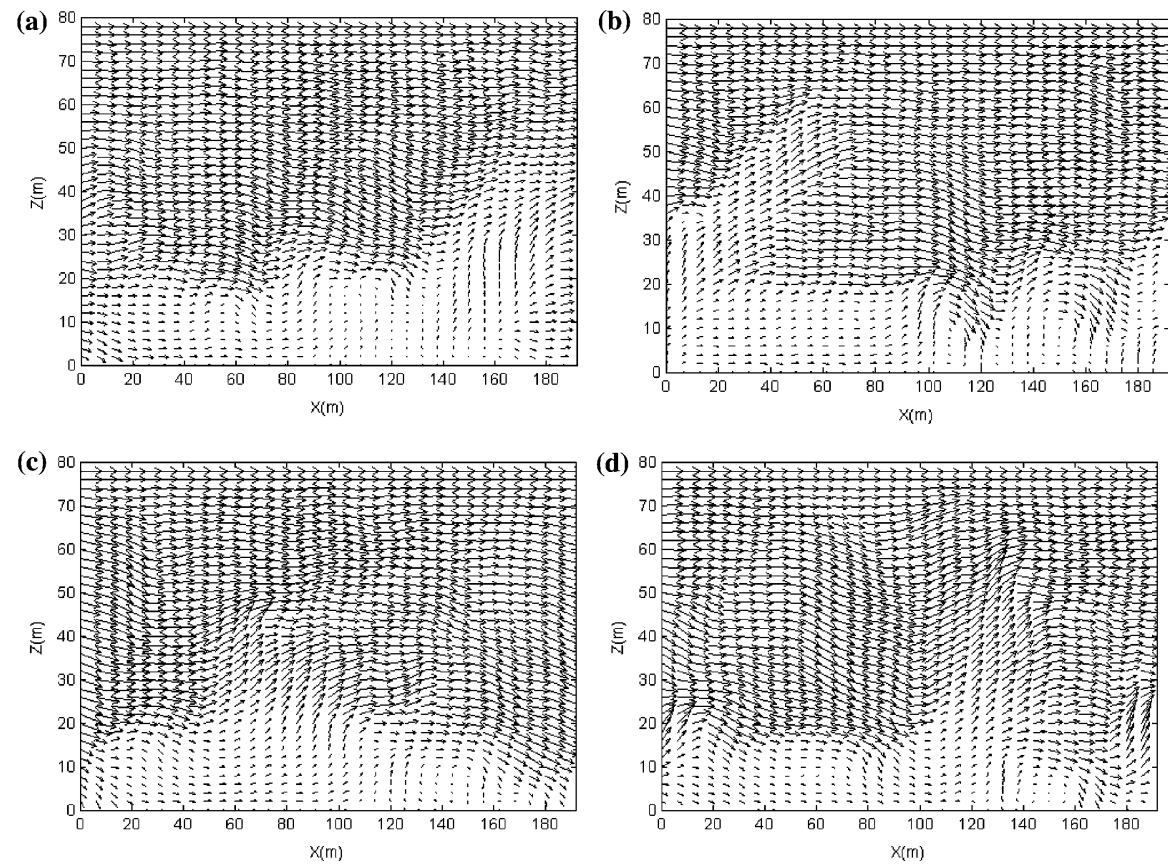

Figure 5. Vertical cross-section of velocity fields at $y=\frac{1}{2} L_{y}$.

The surface layer (SL) of a boundary layer over a rough surface can be subdivided into the inertial sublyer (IS) and the roughness sublayer (RS) [26]. Within the IS, the spatially averaged mean streamwise velocity has an essentially logarithmic profile, and the vertical variation of the shear stress may be neglected $[4,5]$; whereas within the RS, the flow is largely influenced by the individual roughness elements. In the current simulation, we further subdivide the RS for inhomogeneous canopy flows into four regions: (a) canopy front; (b) canopy interior; (c) behind canopy; (d) in front of canopy (see Figure 6).

Figure 7 plots the vertical profiles of laterally averaged streamwise velocity at specified $x$ - locations. In region (a), the vertical profiles of the velocity are adjusted and distorted. Further downstream, it shows an evident inflection point and a reversal of velocity gradient within the canopy. Again as in Figure 5b, a strong upwards flow is found at the leading edge in this region. In region (b), the reversal of velocity gradient remains for a long distance, whereas it finally vanishes in the vicinity of exit of the canopy. However, the inflection point still remains for some distance downstream. The two dashed line profiles are Belcher et al. [1]'s theoretical results in the exit region, which are located at the same stations upstream/downstream of the exit with the corresponding LES profiles. Note Belcher et al. [1] investigated a spatially developing turbulent boundary layer over a step change 
Flow

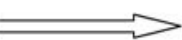

IS

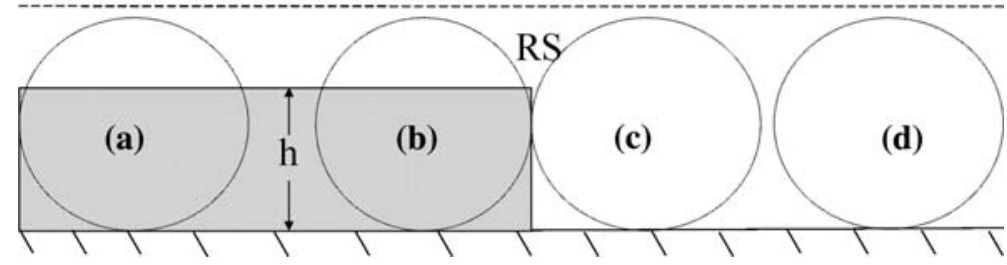

Figure 6. Flow regions. (a)canopy front;(b)canopy interior;(c)behind canopy;(d)in front of canopy.

$\mathrm{X} / \mathrm{h}$

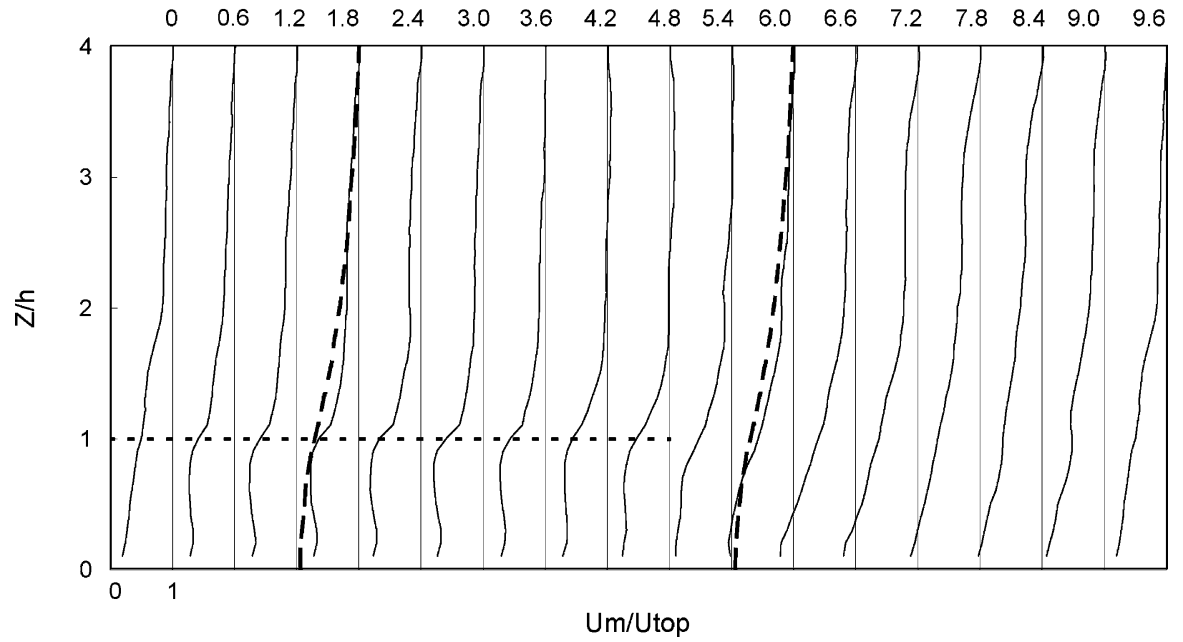

Figure 7. Vertical profiles of laterally averaged streamwise velocity at specified $x$ locations. - LES; - - Belcher et al. [1]'s theoretical data.

of roughness element height, whereas we studied a turbulent flow over an underlying alternately (in streamwise direction) covered with large roughness elements and small roughness elements. In our numerical simulation, periodical boundary condition was imposed both on the inlet-outlet and the lateral boundaries, in which the wake of canopy at outlet was fed back into the flow at inlet. The interaction of the wake with the canopy can not be neglected. In region (c), the flow is fairly weak beneath the top of the canopy. In the front of region (d), the velocity profiles are entirely restored 


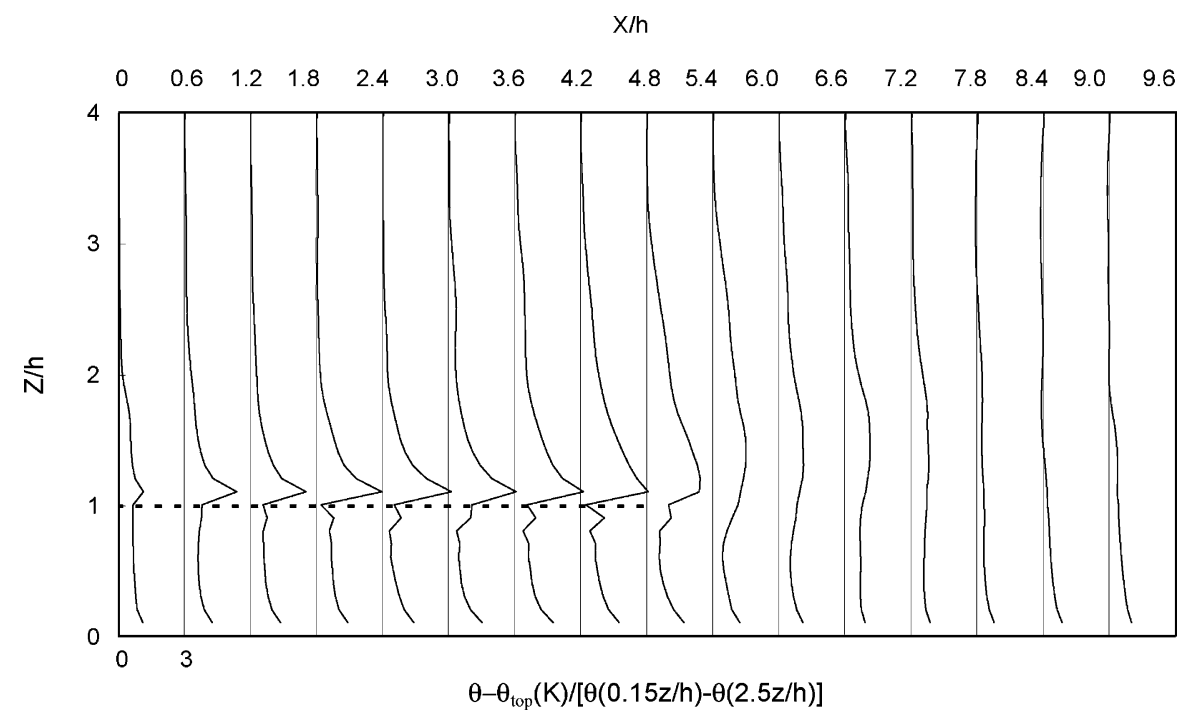

Figure 8. Vertical profiles of laterally averaged temperature at specified $x$-locations, with heat source in plant canopy.

into a near-logarithmic form. Further downstream in the rear of region (d), which is the impact region [1], the velocity profiles start to distort.

Figure 8 plots the vertical profiles of laterally averaged temperature at specified $x$-locations, with heat source in the canopy. The elevated inversion was originally found by field observation above an urban area, e.g. Shenyang, China [27]. It was also successfully simulated by a two-dimensional mesoscale model [20]. In Figure 8, the elevated inversion is evident, which is immediately induced at the leading edge of the canopy, and is enhanced downstream. While further downstream away from the canopy, the thermal plume is dispersed and drifted upwards due to the thermal buoyancy. Note in Figure $1 \mathrm{~b}$ most portion of the heat source for LAI $=5$ is at the top of plant canopy, which is similar to that a scalar is dispersed from an elevated horizontal plane source. In such a case not far downstream from the source, the maximum scalar can be found approximately at the height of the source if there is no other factors, e.g. a thermal buoyancy force, influencing the dispersion. In the current simulation, the thermal buoyancy force is not negligible which drifts upwards the peak of vertical temperature profile. Also note in Figure $5 \mathrm{~b}$ and the corresponding discussion, the upward flows at the leading edge of plant canopy are fairly strong, which brings 'fresh' air mainly into the top area of plant canopy and shifts the peak temperature above the canopy. The domain size in streamwise direction (the width of plant area is only 1.2 times of the depth of the domain,) is not large enough to generate a thicker elevated inversion layer within the 
plant area, even though the mixing of the scalar is very strong due to a strong shear layer above the plant canopy. Nevertheless, the inversion layer gradually becomes thicker and the temperature gap becomes less downstream away from the canopy.

In the current research, we abandoned the attempt to predict accurately the details of the turbulent flow and the temperature transportation within a real urban canopy layer, simulating a group of three dimensoinal obstacles, because it is very complicated and so many processors involved with a broad spectrum of time and spatial scales. We tried to use this very simply model to capture some features in an urban canopy which is adjacent to a green land at night time, where the canopy generates a large heat flux into the atmosphere whereas the green land generates a little heat flux. Also note that the background atmospheric condition in nocturnal ABL is generally stable. Nevertheless, the weakly unstable situation in current LES is worth studying, e.g. for some particular time in the night. The urban heat island (UHI) is evident here (see Figures 5 and 8). Again, because the moisture was not incorporated into the simulation, the UHI effect might be more evident.

The horizontally averaged temperature profile obtained from LES is also compared with the field observation above a horizontally homogeneous forest in a similar condition [10] in Figure 10 (left). Overall, both profiles exhibits a weak unstable condition. It was found that a reduction in rural soil moisture reduces inversion height and increases inversion strength [20]. Martilli further explained that lower rural soil moisture content increases temperatures during daytime (because of stronger sensible heat fluxes), but also increases the strength of the nocturnal stability close to the ground (faster cooling because of a lower heat capacity of drier soil). Since generally the moisture in forest is not low, and the transpiration smooths the temperature gradient within and above the forest canopy, it is not surprising that no elevated inversion was found above the forest canopy in Gao et al.'s field measurements, which again confirms that to some extent the current numerical simulation is alike an urban boundary layer. Nevertheless, the effect of the forest on the temperature profile is quite evident in Gao et al.'s field measurements, which dramatically changes the slope of the temperature profile approximately at the height of canopy.

In order to take account of the transpiration of plant into numerical simulation, we also conducted a simulation imposing heat sink instead of heat source in the canopy. Again, it would be too ambitious for us in the present research to attempt to predict accurately the details of the turbulent flow and the temperature transportation within a real urban canopy layer. We did attempt to use this simply model to capture some features of cool island(CI). Figure 9 plots the vertical profiles of laterally averaged temperature at specified $x$-locations, with heat sink in the canopy. Except 
$\mathrm{X} / \mathrm{h}$

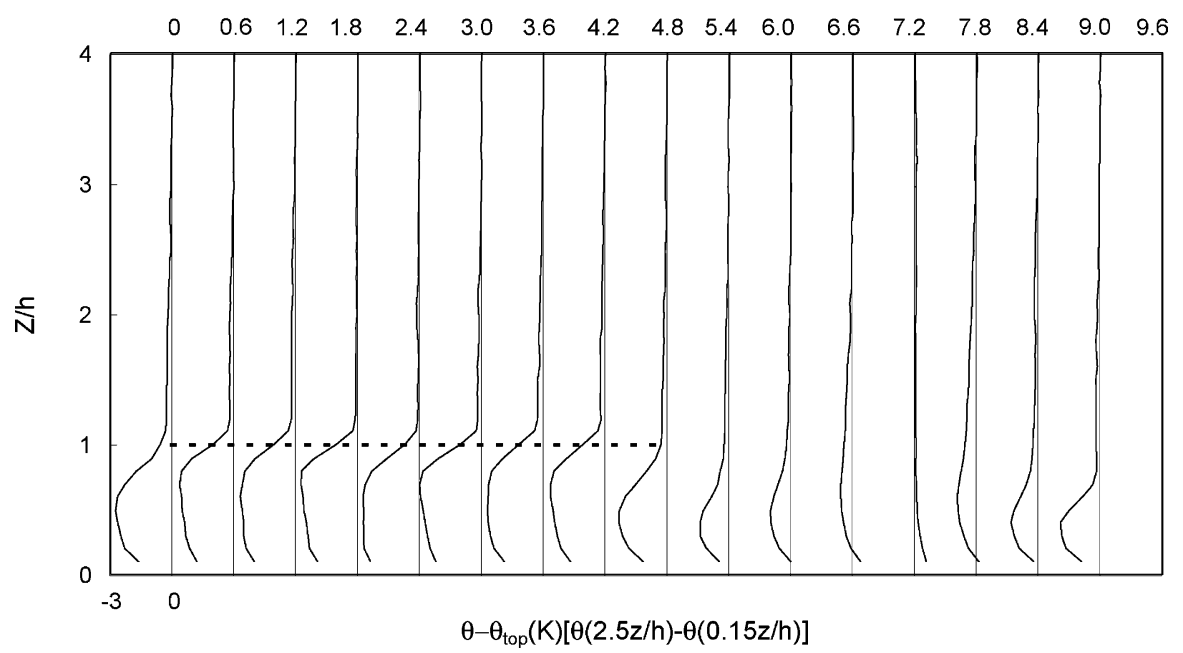

Figure 9. Vertical profiles of laterally averaged temperature at specified $x$-locations, with heat sink in plant canopy.

for heat flux $Q_{*}=-0.084 \mathrm{~ms}^{-1} \mathrm{~K}$ in Figure 9, the other numerical settings are the same as in Figure 8. This case is alike a forest adjacent to a bare soil in weak solar radiation, while the forest with full summer foliage has strong transpiration (see run B in Gao et al. [10]). The CI is evident here. The peak of the profile in the canopy region is located approximately at the same height of the LAI within the canopy as in Figure 1, which is because that the flow within the canopy is fairly stable and an inversion layer exists near the top of the canopy. The depth of the inversion layer keeps almost the same for all the streamwise locations, which differs significantly from that of UHI in figure 8. In region (c), the inversion layer remains for a long distance downstream, gradually decays and finally vanishes. Note in region (d), the influence of the canopy is so dramatical that the inversion is formed far upstream of the leading edge of the canopy. Turning to figure 8 , the elevated inversion is formed only downstream of the leading edge.

Moreover, the horizontally averaged temperature profile obtained from LES is compared with the field measurements above a forest [10] in Figure 10 (right). There might be large scale turbulent structures above the forest in the field measurement, which caused the variation of the temperature with height in Gao et al.'s data. Also note that there is a discrepancy between the measurements and numerical results in the near surface region, which might be due to that the surface condition is slightly different from that in the field measurement. Nevertheless, the numerical results are qualitatively comparable with the field measurements. In particular, both the 

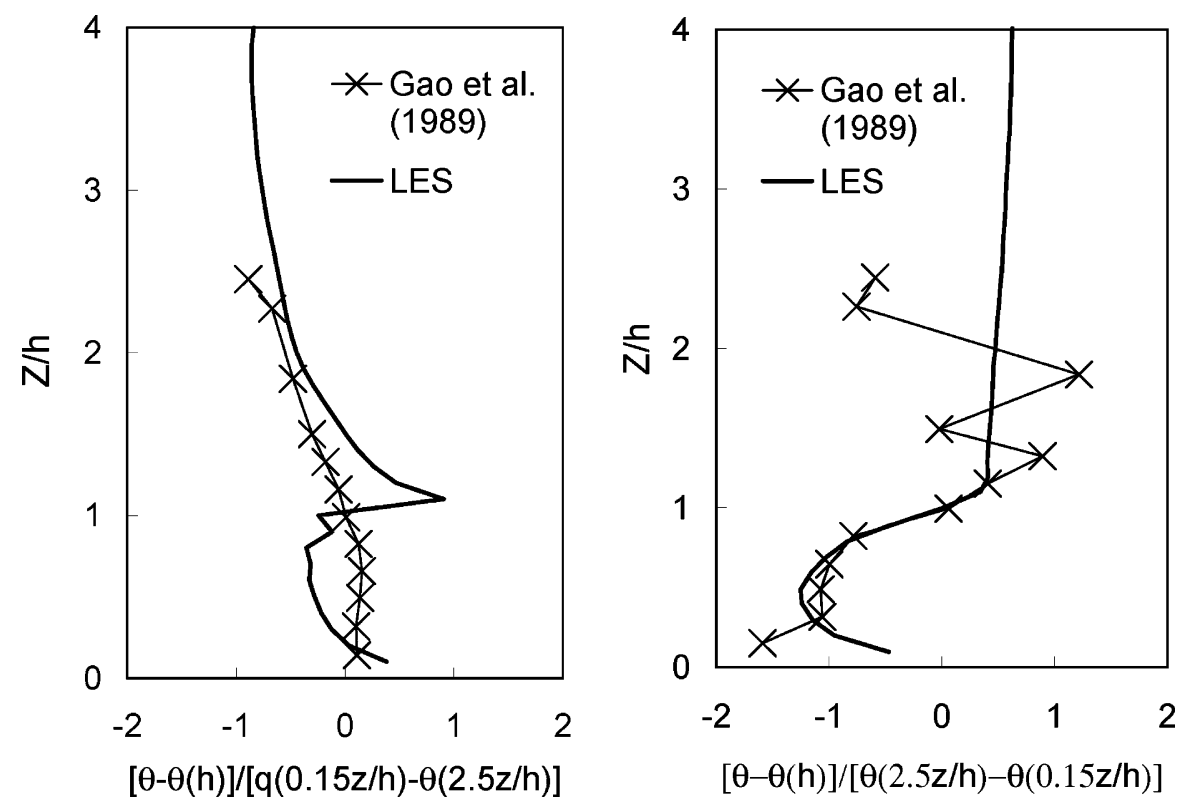

Figure 10. Vertical profiles of horizontally averaged temperature. Left, heat source; right, heat sink.

numerical simulation and the field observation show an evident inversion within the canopy.

\section{Concluding Remarks}

A LES with TSF SGS model, was validated simulating turbulent flow within and above a homogeneous canopy. A simple drag term was added into the momentum equations and a source/sink term was added to the scalar equation to simulate both the aerodynamical and thermodynamical effects of the canopy. The successful validation suggests that the rather coarse mesh can yield quite reasonable results for such flows.

As a start to study the heat, mass and momentum transfer in ABL over inhomogeneous underlying surface, we simulated the turbulent flows over an inhomogeneous canopy, which was alternately (in streamwise direction) large roughness elements $(20 \mathrm{~m}$ height) and small roughness elements (approximately $0.32 \mathrm{~m}$ height).

It was found that the horizontally averaged streamwise velocity over an inhomogeneous canopy was comparable with the flow over a homogeneous canopy with the same horizontally averaged LAI, which suggests that despite the remarkable local difference, it is likely that the horizontally averaged velocity depends predominantly on the average leaf area density of the inhomogeneous canopy with a constant resistance coefficient. 
However, it is known that staggered cubes generate more drag force than aligned cubes with the same plan area density 0.25 and the same mass flux [5]. To calculate such flows using the distributed drag force approach, it is critical to estimate the the resistance coefficient of the obstacles accurately. How to estimate the resistance coefficient in a simple and economical way still remains an issue [19].

We noted that the horizontally averaged streamwise velocity within the canopy in the large roughness region can be larger than that at the same height in the small roughness region. The upward flows at the leading edge of large roughness elements and the weak clockwise circulations in the small roughness region $(z<h)$, which are the shielding of the canopy, may explain this. From the above, it may suggest that some major impacts of inhomogeneity of the roughness elements on the velocity field were successfully captured. Moreover, the adjustment of the simulated velocity over the inhomogeneous canopy was found qualitatively comparable with the theoretical results [1].

To our point of view, UHI is an obvious direct sideeffect of human activity. In some metropolis areas, it may become a serious problem. On the other hand, UHI is likely local(i.e. city scale, see Figure 8), which can be controlled with affordable cost. Using LES, the UHI was successfully reproduced simply imposing heat source in the large roughness region, where an elevated inversion layer was observed over the large roughness elements. But we must stress again using such a simple one-dimensional distributed drag approach can not provide accurate details of the turbulent flow and the temperature transportation within a real urban canopy layer. Furthermore, the CI was also successfully simulated imposing heat sink in the large roughness region. We noted that the features of simulated CI differs significantly with those of simulated UHI, e.g. the location and the depth of inversion layer. New insights into such phenomena have been gained. We conclude that LES provides an efficient tool for the threedimensional inherently unsteady urban-like boundary flows.

\section{Acknowledgements}

The research was supported by the National Natural Science Foundation of China under the grant No. 10332050. The authors are grateful to Drs Chin-Hoh Moeng, and Peter Sulliwan, NCAR, USA for generous permission of using their LES code. The computation was completed using the SGI Origin2000 computer in LASG, Chinese Academy of Sciences. The ZTX is very grateful to Universities Weather Research Network, NCAS for supporting him to continue the research in the same area. The authors would also like to thank the referees for the helpful comments and the suggestions for further study. 


\section{References}

1. Belcher, S.E., Jerram, N. and Hunt, J.C.R.: 2003, Ajustment of a turbulent boundary layer to a canopy of roughness elements, J. Fluid Mech. 488, 369-398.

2. Britter, R.E. and Hanna, S.R.: 2003, Flow and dispersion in urban areas, Annu. Rev. Fluid Mech. 35, 469-496.

3. Castro, I.P.: 2003, CFD for external aerodynamics in the built environment, The QNET-CFD network Newsletter 2:2. 229-259.

4. Cheng, H. and Castro, I.P.: 2002, Near wall flow over urban-like roughness, BoundaryLayer Meteorol. 104, 229-259.

5. Coceal, O., Thomas, T.G., Castro, I.P. and Belcher, S.E.: 2005, Numerical investigation of turbulent flow over cubic roughness arrays, J. Fluid Mech., to be published.

6. Deardorff, J.W.: 1974, Three-dimensional numerical study of the height and mean structure of a heated planetary boundary layer, Boundary-Layer Meteorol. 1, 81-106.

7. Ferziger, J.H.: 1993, Subgrid scale modelling, in Galperin, B. \& Orszag S.A. (eds.) Large eddy simulation of complex engineering and geophysical flows, Cambridge University Press, 1993, 37-54.

8. Finnigan, J.J.: 1979, Turbulence in waving wheat. II Structure of momentum transfer, Boundary-Layer Meteorol. 16, 213-236.

9. Fitzmaurice, L., Shaw, R.H., Paw, U.K.T. and Patton, E.G.: 2003, Three-dimensional scalar microfront systems in a large-eddy simulation of vegetation canopy flow, Boundary-Layer Meteorol. 112, 107-127.

10. Gao, W., Shaw, R.H. and Paw, U.K.T.: 1989, Observation of organized structure in turbulent flow within and above a forest canopy, Boundary-Layer Meteorol. 47, 349377.

11. Horiuti, K.: 1997, A new dynamic two-parameter mixed model for large-eddy simulation, Phys. Fluids 9(11), 3443-3464.

12. Kanda, M. and Hino, M.: 1994, Organized structures in developing turbulent flow within and above a plant canopy, using a large eddy simulation, Boundary-Layer Meteorol. 68, 237-257.

13. Kanda, M., Moriwaki, R. and Kasamatsu, F.: 2004, Large-eddy simulation of turbulent organized structures within and above explicitly resolved cube arrays, BoundaryLayer Meteorol. 112, 343-368.

14. Kline, J.C. and Reynolds, W.C. et al.: 1967, The structure of turbulent boundary layer, J. Fluid Mech. 30, 741-773.

15. Kosovic, B.: 1997, Subgrid-Scale modeling for the large-eddy simulation of high-Reynolds-number boundary layers, em J. Fluid Mech. 336, 151-182.

16. Lesieur, M. and Metais, O.: 1996, New trends in large-eddy simulations of turbulence, Annu. Rev. Fluid Mech. 28, 45-82.

17. Li, J.C. and Ouyang, B. (eds.): 1996, Simulations and Observations of Terrestrial Processes, Science press, Beijing, pp. 121-128.

18. Li, J.C. and Xie, Z.: 1999, large-eddy simulation for canopy turbulent flow, Acta Mechanica Sinica 31(4).

19. Lien, F.-S. and Yee, E.: 2005, Numerical modelling of the turbulent flow developing within and over a 3-D building array, part III: a distirbuted drag force approach, its implementation and application, Boundary-Layer Meteorol. 114, 287-313.

20. Martilli, A.: 2002, Numerical study of urban impact on boundry layer structure: sensitivity to wind speed, urban morphology, and rural soil moisture, J. Appl. Meteor. 41, 1247-1266.

21. Metais, O. and Lesieur, M.: 1992, Spectral large-eddy simulations of isotropic and stably-stratified turbulence, J. Fluid Mech. 239, 157-194. 
22. Moeng, C.-H.: 1984, A large eddy simulation model for the study of planetary boundary layer turbulence, J. Atmos. Sci. 41, 2052-2062.

23. Naot, O. and Mahrer, Y.: 1989, Modelling microclimate environments: a verification study, Boundary-Layer Meteorol. 46, 333-354.

24. Patton, E.G.: 1997, Large Eddy Simulation of Turbulent Flow Above and within a Plant Canopy, Doctoral Dissertation of the Univ. of California, Davis, 1997.

25. Patton, E.G., Shaw, R.H., Judd, M.J. and Raupach, M.R.: 1998, Large eddy simulation of windbreak flow, Boundary-Layer Meteorol. 87, 275-306.

26. Raupach, M.R., Antonia, R.A. and Rajagopalan, S.: 1991, Rough-wall turbulent boundary layers, Appl. Mech. Rev. 44, 1-25.

27. Sang, J., Liu, He., Liu, Hu. and Zhang, Z.: 2000, Observational and numerical studies of wintertime urban boundary layer, J. Wind Eng. Ind. Aerodyn. 87, 243-258.

28. Sagaut, P.: 2001, Large Eddy Simulation for Incompressible Flows, Springer-Verlag, Berlin.

29. Shaw, R.H., den Hartog, G. and Neumann, H.H.: 1988, Influence of foliar density and thermal stability on profiles of Reynolds stress and turbulence intensity in a deciduous forest, Boundary-Layer Meteorol. 45, 391-409.

30. Shaw, R. H. and Schumann, U.: 1992, Large eddy simulation of turbulent flow above and within a forest, Boundary-Layer Meteorol. 61, 47-64.

31. Stoesser, T., Mathey, F., Frohlich, J. and Rodi, W.: 2003, LES of flow over multiple cubes, ERCOFTAC Bulletin No. 56.

32. Thomas, A.S. and Buull, M.K.: 1983, On the role of the wall-pressure fluctuations in deterministic motions in the turbulent boundary layer, J. Fluid Mech. 163, 459-478.

33. Xie, Z. and Li, J.C.: 1997, A model of the land atmosphere interaction with canopy influence, $7 A C M F$, Dec.8-12, Madras, India.

34. Xie, Z., Voke, P.R., Hayden, P. and Robins, G.A.: 2004a, Large-eddy simulation of turbulent flow over a rough surface, Boundary-Layer Meteorol. 111, 417-440.

35. Xie, Z., Hayden, P., Voke, P.R. and Robins, A.G.: 2004, Large-eddy simulation of dispersion: comparison between elevated source and ground level source. J. Turbulence. 5.

36. Xie, Z. and Castro, I.P.: 2005, LES of turbulent flow over wall-mounted cubes using three major CFD codes, School of Engineering Sciences, Technical Report, AFM05/02, University of Southampton.

37. Zang, Y., Street, R.L. and Koseff, J.R.: 1993, A dynamic mixed subgfid-scale model and its application to turbulent recirculation flows, Phys. Fluids 5(12), 3186-3196. 